\title{
斯-
}

\section{La Cooperación Oficial europea y española ante el nuevo escenario del sistema internacional de cooperación al desarrollo}

\author{
Judith Martín Lucas \\ EstudianTE DE Doctorado \\ juditmartin@usal.es \\ David Caballero Franco \\ Profesor Asociado \\ Universidad de Salamanca. Facultad de Educación \\ caballero@usal.es
}

Resumen: A lo largo de la historia la comunidad internacional ha conseguido crear y desarrollar mecanismos e instituciones globales que trabajan en la promoción y gestión de la cooperación para el desarrollo a un nivel institucional. Actualmente, nos encontramos ante una crisis de identidad dentro del sistema de cooperación internacional al desarrollo. Conscientes de ello, las instituciones líderes en este ámbito intentan reorientar las acciones y filosofía que la Cooperación al Desarrollo ha llevado a cabo hasta ahora, es por ello que a lo largo 
de este artículo nos centraremos en la Cooperación Oficial, siendo la que comprende las acciones de carácter público. A través de la Agenda para el Desarrollo Sostenible 2030, se plantean las metas del futuro tras la consecución de los ODM. Ante esta situación pretendemos explicar las características de Europa y España ante esta crisis del sistema y las perspectivas de futuro que ambos deberían tener en cuenta.

Palabras clave: Cooperación, desarrollo, ayuda oficial, Unión Europea, España.

\title{
European and spanish Official Cooperation in light of the new situation in International Development Cooperation system
}

\begin{abstract}
Throughout history the international community has been able to set up and develop mechanisms and global institutions that work to promote and manage cooperation aimed at tackling development at an institutional level. Currently, we are facing an identity crisis within the system of international cooperation for development. Aware of this fact, leading institutions in this sector are also trying to reorient the actions and philosophy that cooperating on development programmes has instigated so far. It is for this reason that we focus throughout this paper on official cooperation, this being the type of cooperation that includes actions of a public nature. Targets for the future are established through the 20130 Agenda for Sustainable Development, once the MDGs have been achieved. This being the situation, we aim to explain the characteristics of Europe and Spain with regard to this systemic crisis and the approaches that both need to adopt in the future.
\end{abstract}

Key Words: Cooperation, development, official aid, European Union, Spain. 


\section{La Cooperación Oficial europea y española ante el nuevo escenario del sistema internacional de cooperación al desarrollo}

Judith

Martín Lucas

David

Caballero Franco

Recibido: 30/06/2016

Aceptado: 10/10/2016

\section{INTRODUCCIÓN. LA COOPERACIÓN OFICIAL AL DESARROLLO.}

La cooperación internacional al desarrollo posee numerosas acepciones, pero podríamos definirla como la acción que comprende actuaciones por actores públicos y privados, que poseen un nivel de renta diferente y cuyo propósito es promover el progreso económico y social de países menos avanzados o en vías de desarrollo con el fin de generar un mundo sostenible (Galan \& Sanahuja, 1999). En este caso, nos centraremos en la Cooperación Oficial, que comprende las actuaciones de carácter público. Dentro del contexto mundial global actual, la comunidad internacional ha conseguido crear y desarrollar mecanismos e instituciones globales que trabajan en la promoción y gestión de la cooperación para el desarrollo a un nivel institucional, de carácter no financiero, como las Naciones Unidas o de carácter financiero como el FMI (Fondo Monetario Internacional), o el BM (Banco Mundial).

Fruto de estas acciones institucionales es la Ayuda Oficial al Desarrollo, AOD en adelante, un importante instrumento de financiación pública que puede ser definida como "los flujos que las agencias oficiales, incluidos los gobiernos estatales y locales, o sus agencias ejecutivas, destinan a los países en desarrollo y a las instituciones multilaterales" según el CAD (Comité de Ayuda 
al Desarrollo). Pero el camino hasta este complejo mecanismo de coordinación y actuación no ha sido fácil, teniendo que sortear dificultades, como la crisis de identidad sufrida tras la Guerra Fría con la llamada "fatiga del donante", que conllevó descensos en los flujos de AOD (Caruncho et. al., 2003). Para superar estos contratiempos, la comunidad internacional consiguió reorientar el sistema internacional de cooperación a través de la celebración de diferentes Cumbres y Conferencias, que parecen tener una aplicación lenta y difícil, en suma, diferentes actuaciones como la Declaración de París, Declaración de Accra o la Ronda de Doha, que aún sigue abierta al debate internacional (Trebucq, 2010; Unceta, Martínez \& Zabala, 2012) ${ }^{1}$. En todas ellas, la Unión Europea ha mostrado una participación activa².

Abriendo la mirada a nuevas perspectivas, la creación del Grupo de Trabajo de Naciones Unidas para coordinar la Agenda del Desarrollo post-2015, como continuación a ODM (Objetivos de desarrollo del Milenio) ${ }^{3}$, generó un gran debate sobre la orientación que debería tomar la AOD, así como el planteamiento de nuevas líneas de acción y trabajo, que han sido y están siendo objeto de críticas formuladas tanto desde posiciones neoliberales como desde los oponentes a la globalización (Sanahuja, 2014). Se presentó la necesidad de trabajar en base a las cuatro dimensiones planteadas en la Declaración del Milenio, económica, política, social y ambiental del desarrollo (Naciones Unidas, 2014) y poner a disposición del cumplimiento de las metas y objetivos todos los recursos disponibles de cada país (Naciones Unidas, 2015), pero se hacía muy difícil desarrollar buenas propuestas si no se preveía una buena coordinación entre los diferentes países. Hasta tal punto, que durante los últimos años se han planteado diferentes aspectos a abordar con el fin de clarificar las políticas de cooperación al desarrollo, como la coherencia de políticas, el debate sobre la financiación y el reto de la gobernanza global

${ }^{1}$ Como bien indican estos autores, entre otros muchos comentarios, la declaración de París, quedó como unos principios para gestionar y distribuir la ayuda, el acuerdo de Accra reconoció la importancia de otros actores y la Ronda de Doha arroja una incógnita a un panorama poco alentador para los países en vías de desarrollo que pretenden abrirse al mercado.

${ }^{2}$ Muestra de ello en: FRIDE. (2008) De París a Accra: Construyendo la Gobernanza Global de la Ayuda. FRIDE 16 1-16.

3 Véase: http://www.undp.org/content/undp/es/home/mdgoverview/mdg goals/post-2015-development-agenda.html (visitado el 25/02/2015). 
(Unceta, 2013 a). Aún así las Naciones Unidas (2014) consideran que tanto la AOD como otros fondos públicos desempeñan un papel central y catalizador, especialmente en los países más vulnerables, así la AOD debería servir tanto para financiar las actividades que hayan podido quedar pendientes en relación con los ODS (Objetivos de Desarrollo del Milenio) como para afrontar la transición y puesta en marcha de la Agenda 2030 para el Desarrollo Sostenible.

Se presenta la necesidad de un cambio estructural, no podemos avanzar hacia una verdadera cooperación internacional si lo que dictamos en un papel en una conferencia en pro del desarrollo, lo deshacemos al mismo tiempo con políticas comerciales, financieras y medioambientales contrarias (Martens, 2013).

\section{EL PAPEL DE LA UNIÓN EUROPEA. UN DONANTE ATÍPICO.}

La Unión Europea, UE de ahora en adelante, comenzó hace décadas participando en el marco de un paradigma clásico Norte-Sur, ateniendo a sus relaciones poscoloniales (Sanahuja, Tezanos, Kern \& Perrotta, 2015) y es un actor que ha mostrado una corta y particular trayectoria, teniendo en cuenta las peculiaridades que caracterizan a esta asociación económica y política.

A día de hoy, se presenta en el CAD (Comité de Ayuda al Desarrollo ${ }^{4}$ como UE, siendo su mayor donante, a la vez, que sus países miembros también forman parte de este comité (En este caso sería Sanahuja et. al, 2015). Pero la UE no es sólo el 28 donante europeo, sino que también actúa como coordinadora, gestora y responsable de políticas ${ }^{5}$. A día de hoy, la UE junto con la comunidad internacional, ha de hacer frente a la crisis de legitimación de la cooperación al desarrollo que vivimos ${ }^{6}$ (Unceta et., al., 2012). Nos encontramos en un momento de inflexión

${ }^{4}$ Véase: Desarrollo y Cooperación. Informe anual 2014 sobre las políticas de la Unión Europea en materia de desarrollo y ayuda exterior y su aplicación en 2013.

http://www.euractiv.es/dossiers/dossier=505 (visitado el 26/02/2014).

${ }^{5}$ Comunicación de la comisión al parlamento Europeo, al consejo, al comité económico y social Europeo y al comité de las regiones (2011) Incremento del impacto de la política de desarrollo de la UE: programa para el Cambio.

${ }^{6} \mathrm{~A}$ lo largo de la primera década del Siglo XXI, la cooperación al desarrollo ha visto mermada la influencia de los flujos de $A O D$, se ha producido un creciente debate sobre la agenda del desarrollo y el cuestionamiento del propio término. 
en el que junto a la globalización financiera se mantiene la necesidad de generar un desarrollo más armónico, equitativo, transparente y sostenible (Unceta, 2013), todo ello ante un contexto de crisis económica mundial que ha dejado patente la volatilidad a la que ha sido sometida la Ayuda Oficial al Desarrollo (García-Arias, 2008). Estas circunstancias ponen en duda el antiguo modelo de ayuda, basado en países donantes que donaban a países receptores.

La UE también ha de tener en cuenta la importancia de nuevos factores emergentes dentro del sistema de cooperación internacional, como son, el auge de la cooperación Sur-Sur, la cooperación triangular o las nuevas políticas de los países emergentes (Robles, 2012; Unceta, 2013; Sanahuja et. al., 2015).

La UE parece haber tenido en cuenta esta construcción de una nueva arquitectura para el desarrollo, así lo muestra en su Programa para el Cambio. Como afirma Granell (2006) una "auténtica política coordinada" de cooperación al desarrollo dentro de la UE, es un deseo ampliamente compartido por todos sus países miembros, pero no es fácil, dado que los diferentes Estados miembros poseen muy diferentes grados de sensibilidad ante esta causa, presentando en un principio, 26 modalidades de sensibilización diferentes alejadas de un consenso, que poco a poco han ido avanzando hacia diversos acuerdos y tratados, permitiendo acercar las posturas de los diferentes estados miembros, de forma interna dentro de la propia Unión y acorde a líneas de actuación que vienen dadas a nivel internacional. Tal es así, que en la actualidad todos los donantes de la UE comparten valores, objetivos de desarrollo, principios y enfoques comunes ${ }^{7}$.

Gracias al Tratado de Lisboa, se ha consolidado una política de desarrollo de la acción exterior de la UE, el SEAE (Servicio Europeo de Acción Exterior) ha demostrado ofrecer ventajas y oportunidades de cara a una cooperación al desarrollo más eficaz. Pero a pesar de que en los tratados, cumbres y conferencias llevadas hasta ahora nos lleva hacia una política de actuación

${ }^{7}$ Comisión de las Comunidades Europeas (2007) Comunicación de la Comisión al consejo y al parlamento europeo. Código de conducta de la UE relativo a la división del trabajo en el ámbito de la política de desarrollo. 
más consensuada y coordinada, el camino no es fácil, así consta en el Programa para el Cambio (2011) ${ }^{8}$. La crisis mundial actual ha hecho más vulnerables a muchos países en desarrollo, algunos países socios han pasado a ser donantes, mientras que otros potenciales han disminuido sus donaciones. Este hecho, ha supuesto que la UE se plantee acciones de complementación y mejora de su política de cooperación al desarrollo acordes y dirigidas a cumplir los principios de la Agenda 2030 para el Desarrollo Sostenible.

\subsection{Relaciones de la Unión Europea y América Latina y Caribe.}

Por otro lado, no podemos olvidar las relaciones que la UE y ALC ( América Latina y Caribe) han mantenido a lo largo de estos años, después de su larga trayectoria de diálogo político y cooperación birregional y a 16 años de la celebración de su primera cumbre birregional, se encuentran en un momento de balance y cambio de ciclo (Sanahuja, 2015). Esta asociación estratégica que mantienen la UE y ALC (América Latina y Caribe) puede jugar un papel importante en materia política en la puesta en marcha y desarrollo de la Agenda para el Desarrollo Sostenible 2030 (Sanahuja et, al., 2015). Un ejemplo de ello es el programa EUROsociAL, el más representativo para promover la cohesión social, aprendizaje entre pares y el intercambio de experiencias entre ambas regiones ${ }^{9}$. Los buenos resultados de este programa han hecho que se renueve a una tercera fase, EUROsociAL+ 2016-2021, centrada en modificar políticas sociales, de gobernanza e igualdad de género ${ }^{10}$. No obstante, entre 2015 y 2020 será necesario revisar el modelo de relación de la UE con los países en desarrollo, y en particular su política de cooperación (Sanahuja, 2015).

${ }^{8}$ Comunicación de la comisión al parlamento Europeo, al consejo, al comité económico y social Europeo y al comité de las regiones (2011) Incremento del impacto de la política de desarrollo de la UE: programa para el Cambio.

${ }^{9}$ EUROsociAL. Programa para la Cohesión Social en América Latina: http:// eurosocial-ii.eu/es/pagina/el-programa (visitado el 09/09/2016).

${ }^{10}$ EUROsociAL+: nueva fase del programa de la Unión Europea para incrementar la cohesión en América Latina: http://eurosocial-ii.eu/es/noticia/eurosocial-puesta-en-marcha-de-la-nueva-fase-del-programa-de-la-1 (visitado el 09/09/2016) 


\subsection{El Programa para el Cambio.}

Dentro del ya citado Programa para el Cambio, se pretende, hacer frente a los tres frentes mencionados anteriormente, la coherencia de políticas, la financiación ${ }^{11}$ y el reto de la gobernanza global. Para ello, la UE fomenta un diálogo a escala nacional en un marco de coordinación de los diferentes donantes. Un potente mecanismo que permitirá generar coherencia dentro de los diferentes actores de la UE es la complementariedad, que responde a la coordinación de la ayuda, "implica que cada actor centre la ayuda en los ámbitos en los que aporte mayor valor añadido"12. Una buena complementariedad de la ayuda, generará una disminución en los costes administrativos, un trabajo eficiente y eficaz, a través de concentrar la ayuda de los países donantes en un máximo de tres sectores por país (Robles,2012) atendiendo a aquellos conocimientos técnicos y específicos que mejor puedan complementar a los países receptores, porque la Cooperación al Desarrollo no es solo en términos monetarios, va mucho más allá. No obstante, dentro de este programa la UE ha de hacer frente a ciertas incoherencias que hacen referencia a sus políticas comerciales o de desarrollo, debido a decisiones tomadas en marcos distintos y anteriores a los objetivos post-2015, entre ellas, la graduación que contempla el Programa para el Cambio (Sanahuja, 2015).

\subsection{Perspectivas de futuro.}

La UE ha sido un impulsor activo e implementador de los consensos multilaterales de desarrollo (Sanahuja et al., 2015). Presentándose como un potente organismo de coordinación de políticas de Cooperación al Desarrollo y un donante consolidado y extendido en los países del Sur, debe tomar parte y responsabilidad en el papel de la construcción y toma de decisiones en la Agenda 2030 para el Desarrollo Sostenible, siendo esta una de

${ }^{11}$ El cambio en la financiación estaría en línea con los acuerdos llevados a cabo en Busán, en 2011. Para más información véase: http://www.euractiv.es/ dossiers/dossier $=505$ (visitado el 26/02/2016).

http://www.effectivecooperation.org/files/OUTCOME_DOCUMENT-FINAL_ ES.pdf (visitado el 26/02/2016).

${ }^{12}$ Comisión de las Comunidades Europeas (2007) Comunicación de la Comisión al consejo y al parlamento europeo. Código de conducta de la UE relativo a la división del trabajo en el ámbito de la política de desarrollo. 
las iniciativas más importantes e influyentes promovidas por la comunidad internacional en el último siglo, cuya pretensión es asegurar que la globalización se convierta en un proceso positivo para todo el mundo ${ }^{13}$. La UE deberá trabajar en un nuevo contexto, en el que se hace necesario tener en cuenta el doble rol de los países de renta media, países emergentes y en vías de desarrollo. Deberá abordar, de manera interna y junto con la comunidad internacional aquellas críticas, resultados y consecuencias derivados de los ODM (Objetivos del Milenio).

Además, debe trabajar por la promoción de la sostenibilidad, superando los inconvenientes que presentó este principio en los ODM (Objetivos del Milenio) y abogando junto con este eje, la lucha contra la pobreza extrema en el mundo, que viene de la mano de la lucha contra las desigualdades y hacer frente a unos medios de financiación suficientes y predecibles (Alonso, 2013). Un problema que ha tocado de lleno a la UE en la última década a causa de la crisis económica, poniendo de manifiesto, por un lado, la gran responsabilidad de esta institución en la cooperación al desarrollo, así como las diferentes tendencias entre sus donantes a pesar de la crisis, países como Holanda han bajado por primera vez del 0,7, mientras que otros como Reino Unido lo ha alcanzado, a pesar de las complicadas circunstancias. Un ejemplo de gran retroceso en este sentido es España, un país que ha presentado un dramático descenso siendo en el pasado uno de los países más activos en cuanto a la escena internacional de la Cooperación al Desarrollo (Sotillo, 2012; Oxfam, 2013). Este marco organizativo y principios rectores de la Cooperación al Desarrollo en la UE y su papel en la Agenda 2030 para el Desarrollo Sostenible afectan a la política de Desarrollo Española, que está pasando, en el momento actual, por un grave periodo de crisis.

\section{ESPAÑA. HACIENDO FRENTE A UNA GRAVE CRISIS DE LA COOPERACIÓN.}

Según la Comisión Europea (2013) y Naciones Unidas (2014) la responsabilidad para alcanzar los resultados perseguidos es en primer lugar y ante todo, nacional. Para poder llevar a cabo

13 Véase: http://www.un.org/en/development/desa/policy/untaskteam_undf/ executive\%20summary spanish.pdf (visitado el 26/02/2016). 
una gobernanza eficaz para el desarrollo sostenible las instituciones públicas de todos los países han de ser inclusivas y rendir cuentas a la población de sus acciones, y este contexto se reconoce como papel fundamental el que cumple la AOD (Naciones Unidas, 2014; Comisión Europea, 2014) y que ha sido fuertemente castigada a lo largo de los últimos años. El esfuerzo relativo en materia de AOD cayó un $70 \%$ entre 2009 , en el que representaba el 0,46\% del PIB (Producto Interior Bruto) y el 2015, en el que pasó a representar tan solo el 0,13\% del PIB. Estos recortes amenazan los programas de prestigio construidos en regiones como América Latina y África, además de difuminar el papel de España en la comunidad de donantes (Oxfam Intermón, Unicef, 2016).

Es cierto que la política española de cooperación para el desarrollo deberá hacer frente a un contexto de recursos limitados (Robles, 2012), y parece difícil que con los enormes recortes presupuestarios en materia de desarrollo pueda alcanzar la meta 17.2 de la Agenda 2030 que compromete a los países desarrollados a destinar el 0,7\% del PNB (Producto Nacional Bruto), a la AOD además del compromiso y posición en relación a la ayuda europea (Pérez, 2016). Pero ello no ha de servir de excusa para no seguir trabajando en pro de una mejor calidad, eficacia y adecuación de la ayuda, trabajando de forma acorde y complementaria a los nuevos cambios propiciados y promovidos tanto en el seno de la UE como en la comunidad internacional.

A la hora de planificar el cumplimiento de sus compromisos, España debe determinar y aclarar en qué medida se trata de compromisos nacionales y en qué medida son una obligación que comparte con el resto de socios Europeos (Pérez, 2016).

El estado español, ha de configurar una posición dentro del Grupo abierto de los ODS (Objetivo de Desarrollo Sotenible) ${ }^{14}$, y por ende, en la construcción de una posición para la Agenda 2030 para el Desarrollo Sostenible. La participación de España en esta agenda, implica no solo movilizar presupuestos, instituciones y normas nacionales que deberán ir siempre acordes con la política de la UE (Pérez, 2016). Es importante que España

${ }^{14}$ Plan Director de la Cooperación Española 2013-2016: http://www.cooperacionespanola.es/sites/default/files/plan_director_cooperacion_espanola 2013-2016.pdf (visitado el 26/02/2016). 
tome un papel de responsabilidad y participe activamente en el desarrollo y puesta en marcha de esta Agenda, ya que, esta misma deber ser concebida como una Agenda global, en la que todos los países compartan responsabilidades ${ }^{15}$. A pesar de ello, un año después de la firma de los Objetivos de DesarroIlo Sostenibles, España sigue sin definir ninguna medida que permita tan siquiera ver una hoja de ruta para construir, aplicar y monitorear las estrategias que permitan alcanzar los 17 objetivos y las 169 metas del acuerdo global (Oxfam Intermón, Unicef, 2016).

El único aspecto positivo en este sentido, es que España hasta el momento ha mantenido un rol activo dentro de los debates realizados en esta Agenda, comprometiendo la dotación del primer fondo para los ODS (Objetivos de Desarrollo Sostenible) (Oxfam, 2015).

\subsection{La Agenda para el Cambio y la Programación Conjunta.}

A pesar del ínfimo presupuesto al que ha quedado configurada la Cooperación Oficial al Desarrollo en España, se esta luchando y trabajando por generar mecanismos acordes con la UE, es por ello, que España siguiendo las directrices marcadas por las Naciones Unidas (2014) se focalizará en una serie de regiones prioritarias y tomará en cuenta a la UE como un importante organismo dentro de la cooperación multilateral, dentro de la Agenda para el Cambio, citada con anterioridad, España trabajará a través de mecanismos como la Programación Conjunta, con el fin de reducir la fragmentación de la ayuda y colaborar a que la ayuda tenga un mayor y mejor impacto ${ }^{16}$ y la Cooperación Delegada, a través de la cual, un donante actúa en nombre de uno o varios donantes $^{17}$ (Robles, 2012). Algunos de estos pasos ya han sido dados, España ha conseguido reducir su dispersión geográfica de AOD, y pretende concentrar su ayuda a un sector estratégico como es América Latina (Oxfam, 2013 a). Basta con acudir al

\footnotetext{
${ }^{15}$ Ibidem

${ }^{16}$ Comunicación de la comisión al parlamento Europeo, al consejo, al comité económico y social Europeo y al comité de las regiones (2011) Incremento del impacto de la política de desarrollo de la UE: programa para el Cambio.

${ }^{17}$ Comisión de las Comunidades Europeas (2007) Comunicación de la Comisión al consejo y al parlamento europeo. Código de conducta de la UE relativo a la división del trabajo en el ámbito de la política de desarrollo.
} 
mapa de actuación de la AECID y de Europe Aid para comprobar los avances en este terreno ${ }^{18}$.

\subsection{Las autonomías y la Cooperación al Desarrollo en España.}

Por último, España ha de hacer frente a otra disyuntiva que se presenta de la mano de las autonomías. Las partidas que las Comunidades Autónomas destinan a Cooperación al Desarrollo han sido en la última década, muy variables. A pesar de presentar diecisiete modelos de Cooperación al Desarrollo muy similares, sus partidas presupuestarias son muy distantes, solamente Euskadi, contribuyó en el año 2012 a la tercera parte del total de la AOD canalizada a través de las Comunidades Autónomas (Oxfam 2013;2015). Es difícil trabajar en el plano internacional, cuando de forma interna nuestra Cooperación al Desarrollo presenta grandes disparidades. ¿Deben perder o retomar protagonismo las Comunidades Autónomas en la política de Cooperación al Desarrollo Española? La respuesta puede ser objeto de gran debate, pero lo que está claro es que la Cooperación al Desarrollo debería considerarse una política seria y estable, que no cambie dentro de una autonomía en función de las preferencias de esta o el gobierno que dirija la acción en esos momentos. Las Comunidades Autónomas, en mayor medida, junto con Ayuntamientos y Universidades, presentan en nuestro país un potente mecanismo para dar a conocer todos los y procesos que se están llevando a cabo en la Cooperación Española y Europea de cara al planteamiento del desarrollo post 2015, no podemos permitir el debilitamiento de este sistema que cada vez se ve más dañado debido a los grandes recortes presupuestarios y el periodo de incertidumbre política que vivimos en nuestro país actualmente.

\subsection{Un futuro de incertidumbre.}

A pesar de los esfuerzos y el trabajo llevado a cabo, España presenta grandes desventajas e inconvenientes que ponen freno al nuevo despegue de sus políticas de cooperación al desarrollo y su participación en la Agenda 2030 para el Desarrollo Sostenible,

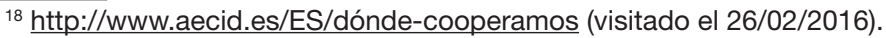

https://ec.europa.eu/europeaid/countries-territories-and-regions-where-weare-active en (visitado el 26/02/2016).
} 
por un lado, en el plano interno, se hace necesaria una revisión del FONPRODE (Fondo para la Promoción del Desarrollo), más allá de su reforma "clandestina" (Oxfam, 2013; 2015).

Por otro lado, ha perdido protagonismo en la toma de decisiones de carácter internacional, la disminución de su ayuda ha limitado su capacidad operativa, y así, la participación activa en el ámbito internacional. Finalmente, se presenta necesario establecer y fijar los presupuestos desde una lógica a largo plazo, una política y mecanismo tan importante como es la Cooperación al Desarrollo no puede verse cada año en la incertidumbre de ser sometido o no a un gran recorte presupuestario, cuyas consecuencias más severas, recaen en las personas más vulnerables. A la vez que ha de trabajar en la participación, propuestas y puesta en marcha de la Agenda 2030 para el Desarrollo Sostenible, ha de recuperar el papel destacado que la AECID poseía, y así, recuperar el perfil internacional (Oxfam, 2013). En este sentido, la Agenda 2030 para el Desarrollo Sostenible, supone un cambio de paradigma y una oportunidad que el conjunto del gobierno debería aprovechar para reestructurar el diseño de sus políticas públicas (Oxfam Intermon, Unicef, 2016).

\section{CONCLUSIONES.}

En definitiva, el mundo en el que vivimos se presenta con avances extraordinarios frente a niveles inaceptables de desigualdad, la necesidad de retomar el proceso de globalización hacia un camino positivo es cada vez mayor. Como se menciona en "En el camino hacia la dignidad para 2030" tenemos los conocimientos y medios necesarios para hacer frente a las necesidades.

Las instituciones públicas deben liderar un nuevo camino, la construcción de una nueva Agenda 2030 para el Desarrollo Sostenible puede ser el principio. Esta agenda puede y debe representar un pacto global para el desarrollo, o como afirma Sanahuja (2014) un marco multilateral más representativo y legítimo. Debemos dirigir la acción internacional hacia el desarrollo, no permitir que instrumentos como las finanzas generen grandes beneficios para unos pocos, parece ser que en este sentido se está avanzando poco a poco, pero aún queda mucho por hacer. Y para ello se debe llevar a cabo una reforma en los mecanismos 
de gobernanza mundial y han de establecerse lazos y alianzas eficaces para un desarrollo sostenible. Se precisa de una reforma estructural dentro del sistema, no podemos avanzar si lo que acordamos en un papel lo deshacemos por otro lado con políticas medioambientales, financieras o comerciales contrarias.

La UE como defensora de la Alianza Mundial y con la responsabilidad que tiene como gran potencia, debería ser una de las encargadas de movilizar la acción a todos los niveles, incluyendo el sector privado, la sociedad civil, las instituciones científicas y del conocimiento, parlamentos y administraciones locales (Comisión Europea, 2014) y hacer frente a la crisis de legitimación de la cooperación al desarrollo que vivimos. Sin duda alguna, la Unión Europea, junto con sus 28 países donantes, tiene una gran responsabilidad en este proceso, siendo el mayor donante del CAD (Comité de Ayuda al Desarrollo), se hace necesario, por un lado, una mayor homogeneización de las políticas de desarrollo dentro de sus países miembros, además de impedir la volatilidad que la AOD ha sufrido hasta ahora en función de los ciclos económicos. Ante un momento en el que los principales donantes están pasando por una etapa de crisis, es importante tener en cuenta instrumentos nuevos o complementarios a la AOD (Comisión Europea, 2014) , como se menciona en la alianza de Busán (Robles, 2012), que no comprometan el proceso de desarrollo de aquellos países que necesitan de estos instrumentos y alianzas para poder avanzar. La AOD es un importante instrumento de financiación pública para el desarrollo, pero no debe ser el único.

Se hace necesario que el debate sobre la cooperación se centre en un análisis integral del estado de la cuestión que vaya más allá de la ayuda, la complementariedad y coherencia de políticas que se cumplan a través de acuerdos y tratados vinculantes, y la incorporación de mecanismos y agentes nuevos y complementarios al sistema actual (Unceta et. al., 2012). Es importante que se forje un nuevo consenso, más global, más participativo, tanto España como la Unión Europea han de trabajar para que los gobiernos receptores de ayuda se conviertan en gobiernos autónomos, capaces de gestionar y no solo de ser meros receptores pasivos al cumplimiento de las ordenes de un donante. Las perspectivas de la Agenda 2030 para el Desarrollo Sostenible parecen claramente vincularse a la gobernanza global, no parece coherente que ante un mundo global, la Cooperación al Desarrollo se lleve de forma 
descentralizada y desorganizada por parte de todos los países, debemos trabajar para que los instrumentos públicos tengan un efecto positivo en aquellos que son más vulnerables. La ayuda puede ser un gran contribuyente del buen gobierno, que abogue por el interés común, fortaleciendo mecanismos de control y estableciendo mecanismos equitativos para poder afrontar las reglas del juego globales ante las que las diferentes naciones se mueven a día de hoy.

La falta de voluntad en la agenda política en nuestro país es un hecho más que evidente a día de hoy. En concreto, no podemos permitir que en un país como España, la dotación presupuestaria en materia de cooperación al desarrollo esté sujeta a una incertidumbre de un año para otro, y menos aún, ser uno de los países que mayor retroceso a presentado en este ámbito.

La próxima elaboración del V Plan Director de la Cooperación Española se presenta como una oportunidad idónea para contemplar todos estas reflexiones y debates sobre la política española de desarrollo, así como para integrar la dimensión europea. El impulso que necesita la cooperación española a partir de ahora, debe darse no sólo desde las agencias de cooperación, sino que debe contar con el impulso de todas las administraciones afectadas (Oxfam Intermon, Unicef, 2016).

Por último, se presenta en la comunidad internacional, la necesidad de trabajar por una nueva arquitectura financiera, partiendo del supuesto de que las finanzas internacionales deberían ser una de las herramientas más adecuadas para potenciar el desarrollo, se han planteado numerosos mecanismos y alternativas públicas de recaudación de impuestos que vayan dirigidos hacia la Cooperación al Desarrollo, algunos de ellos pueden ser la gran conocida Tasa Tobbin o la Tasa Solidaria sobre los Billetes de Avión (García-Arias, 2013). Es evidente que estos mecanismos deben cumplir requisitos fundamentales como la rendición de cuentas, la transparencia y la revisión efectiva de los progresos hechos.

\section{BIBLIOGRAFÍA}

AECID (2016). Dónde cooperamos. http://www.aecid.es/ES/dónde-cooperamos (visitado el 26/02/2016). 
Alonso, J.A. (2013). Algunas consideraciones sobre la Agenda Post-2015. en La Agenda al Desarrollo Post- 2015: ¿Más de lo mismo o el principio de la transición? Economistas Sin Fronteras.

Asamblea General de Naciones Unidas. (2014). El camino hacia la dignidad para 2030: acabar con la pobreza y transformar vidas protegiendo el planeta.

Caruncho, M., Mestre, C., Miranda, F., Santos, R. (2003). Avances y retos en el sistema de cooperación al desarrollo. GlobalHoy. (10-11) 26-31.

Comisión Europea (2013). Una vida digna para todos. Acabar con la pobreza y dar al mundo un futuro sostenible. Comunicación de la CE [COM(2013)92]: http://eur-lex.europa.eu/LexUriServ/ LexUriServ.do?uri=COM:2013:0092:FIN:es:PDF (Visitado el 19/09/2016).

Comisión Europea (2014). Una vida digna para todos: de la visión a la acción colectiva. Comunicación de la CE [COM(2014)335] Bruselas: http://ec.europa.eu/transparency/ regdoc/rep/1/2014/ES/1-2014-335-ES-F1-1.Pdf (visitado el 16/09/2016).

Comisión de las Comunidades Europeas (2007). Comunicación de la Comisión al consejo y al parlamento europeo. Código de conducta de la UE relativo a la división del trabajo en el ámbito de la política de desarrollo.

Comunicación de la comisión al parlamento Europeo, al consejo, al comité económico y social Europeo y al comité de las regiones (2011). Incremento del impacto de la política de desarrollo de la UE: programa para el Cambio.

Cuarto foro de alto nivel sobre la eficacia de la ayuda. Busan 2011. http://www.effectivecooperation.org/files/OUTCOME DOCUMENT-FINAL ES.pdf (visitado el 26/02/2016).

Galan, M. y Sanahuja, J.A. (1999). El sistema internacional de cooperación al desarrollo, Madrid, CIDEAL.

García-Arias, J. (2008). Finanzas internacionales y desarrollo: ¿oportunidad o amenaza? Revista de la CEPAL (96) 157-172.

García Arias, J.,Vázquez, A., Fernández-Huerga, E. (2013). Financiación Innovadora para el Desarrollo: ¿un deux ex machina 
para financiar la agenda del desarrollo post-2015? Revista de Economía Mundial 36 49-70.

Desarrollo y Cooperación. Informe anual 2014 sobre las políticas de la Unión Europea en materia de desarrollo y ayuda exterior y su aplicación en 2013. (2014). Comisión Europea.

European Comision (2015). Europe Aid. Countries, territories and regios where we are active.https://ec.europa.eu/europeaid/ countries-territories-and-regions-where-we-are-active en (visitado el 26/02/2016).

Diccionario Hegoa: http://www.dicc.hegoa.ehu.es/listar/mostrar/44 (visitado el 24/02/2016).

EUROsociAL. Programa para la Cohesión Social en América Latina: http://eurosocial-ii.eu/es/pagina/el-programa (visitado el 09/09/2016).

EUROsociAL+: nueva fase del programa de la Unión Europea para incrementar la cohesión en América Latina: http://eurosocial-ii.eu/es/noticia/eurosocial-puesta-en-marcha-de-lanueva-fase-del-programa-de-la-1 (visitado el 09/09/2016).

FRIDE. (2008). De París a Accra: Construyendo la Gobernanza Global de la Ayuda. FRIDE 16 1-16.

Martens, J. (2013). Hacía un marco de objetivos universales de sostenibilidad como parte de una agenda Post-2015, en La Agenda al Desarrollo Post- 2015: ¿Más de lo mismo o el principio de la transición? Economistas Sin Fronteras.

Naciones Unidas (2014). Objetivos de Desarrollo del Milenio. Informe de 2014. Nueva York: http://www.un.org/es/millenniumgoals/pdf/mdg-report-2014-spanish.pdf (Visitado el 18/09/2016)

Naciones Unidas (2015). Transformar nuestro mundo: la agenda 2030 para el Desarrollo Sostenible. Resolución aprobada por la Asamblea General el 25 de septiembre de 2015. Nueva York. Naciones Unidas: http://www.un.org/ga/search/view doc.asp?symbol=A/70/L.1\&Lang=S (Visitado el 15/09/2016)

Naciones Unidas (2016). El futuro que queremos para todos. http:// www.un.org/en/development/desa/policy/untaskteam_undf/ executive\%20summary spanish.pdf (visitado el 26/02/2016).

Oxfam (2013). Spain is different. "La realidad de la ayuda 2013". 
Oxfam (2013 a). La ayuda oficial al desarrollo en España. La realidad de la ayuda 2013. Real Instituto Elcano:

http://www.realinstitutoelcano.org/wps/portal/web/rielcano es/ contenido?WCM GLOBAL CONTEXT=/elcano/elcano es/ zonas_es/ari36-2016-perez-espana-y-la-ue-ante-la-agenda2030-quien-hace-que (visitado el 20/09/2016).

Oxfam Intermon (2015). 2015: Año cero.

Oxfam Intermón, Unicef. (2016). Un año de Agenda 2030 en España. Mucho por hacer: https://www.unicef.es/sites/www.unicef. es/files/folleto 12pg uni oxfam.pdf (visitado el 30/09/2016)

Pérez, A. (2016). España y la UE ante la Agenda 2030: ¿quién hace qué?

Plan Director de la Cooperación Española 2013-2016: http:// www.cooperacionespanola.es/sites/default/files/plan director cooperacion espanola 2013-2016.pdf (visitado el 26/02/2016).

Robles, G. (2012). Una nueva política de cooperación para tiempo de crisis. Tiempo de paz. 105 21-26.

Sanahuja, J. A. (2014). De los Objetivos del Milenio al desarrollo sostenible: Naciones Unidas y las metas globales post-2015. En Mesa, M. (2014). Focos de tensión, cambio geopolítico y agenda global. Anuario 2014-2015. Madrid. Centro de Educación e Investigación para la Paz (CEIPAZ). pp 49-83.

Sanahuja, J. A. (2015). La UE y CELAC: revitalización de una relación estratégica. Hamburgo. Fundación EU-LAC.

Sanahuja, J. A., Tezanos, S., Kern, A., Perrotta, D. (2015). Más allá de 2015: Perspectivas y propuestas para la cooperación al desarrollo entre la Unión Europea y América Latina y el Caribe. Hamburgo: Fundación EU-LAC.

Sotillo, J. A. (2012). La cooperación española en la unidad de cuidados intensivos. Tiempos de paz. 105 5-14.

Sustainable Development Goals ( SDGs) http://www.undp.org/ content/undp/en/home/sdgoverview/post-2015-development-agenda.html (visitado el 25/02/2016).

Trebucq, F. (2010). La Ronda de Doha ante la Posibilidad del Fracaso. GEIC 2-9. 
Unceta, K. (2013). Cooperación para el desarrollo: Anatomía de una crisis. Íconos. 47 15-29.

Unceta, K. (2013 a). Más allá del 2015: el debate sobre la agenda de desarrollo y la cooperación internacional. Revista española de desarrollo y cooperación. 33 125-136.

Unceta, K., Martínez, M.J., Zabala, I. (2012). Nueva York, Monterrey y París: tres lógicas contradictorias para enfrentar los retos del desarrollo en la primera década del siglo. Revista Iberoamericana de Estudios de Desarrollo. 1 100-116.

http://www.euractiv.es/dossiers/dossier $=505$ (visitado el 26/02/2016). 